\title{
ALGEBRAICALLY DEPENDENT FUNCTIONS OF A COMPLEX AND $p$-ADIC VARIABLE
}

\author{
D. L. HILLIKER
}

A set of functions $f_{1}(z), \cdots, f_{m}(z)$ are algebraically dependent if there is a polynomial $P\left(w_{1}, \cdots, w_{m}\right)$ with complex coefficients for which $P\left(f_{1}(z), \cdots, f_{m}(z)\right) \equiv 0$. In a previous paper (Hilliker [2]) conditions were established under which $m$ functions $f_{1}(z), \cdots, f_{m}(z)$ of a complex variable $z$ are algebraically dependent. It was assumed that $f_{1}(z), \cdots, f_{m}(z)$ are analytic at one point and that the functional values $f_{\mu}\left(z_{\nu}\right)(\mu=1,2, \cdots, m ; \nu=1,2,3, \cdots)$ at a convergent sequence of distinct points $\left\{z_{\nu}\right\}$ are algebraic. Let $H\left(f_{\mu}\left(z_{\nu}\right)\right)$ denote the height of the algebraic number $f_{\mu}\left(z_{v}\right)$; that is, the maximum of the absolute values of the (integral) coefficients of the minimal polynomial for $f_{\mu}\left(z_{\nu}\right)$. Let $\operatorname{deg} f_{\mu}\left(z_{\nu}\right)$ be the degree of $f_{\mu}\left(z_{\nu}\right)$. Then, roughly speaking, if $H\left(f_{\mu}\left(z_{\nu}\right)\right)$ and $\operatorname{deg} f_{\mu}\left(z_{\nu}\right)$ for $\mu=1, \cdots, m$ and $\nu=\nu_{0}+1, \nu_{0}$ $+2, \cdots$, are not too large and if the sequence $\left\{z_{\nu}\right\}$ converges sufficiently rapidly, $f_{\mathbf{1}}(z), \cdots, f_{m}(z)$ are algebraically dependent.

In this paper we wish to relax the restriction on the size of $H\left(f_{\mu}\left(z_{v}\right)\right)$ and $\operatorname{deg} f_{\mu}\left(z_{\nu}\right)$ and on the rate of convergence of $\left\{z_{\nu}\right\}$. This is accomplished by assuming that the functions $f_{1}(z), \cdots, f_{m}(z)$ are simultaneously analytic in the complex and $p$-adic sense at some point. All of the theorems concerning algebraicness and algebraic dependence in the paper Hilliker [2] lend themselves to $p$-adic generalizations. To illustrate the type of extension that is possible we shall generalize Theorems IV and VII.

Let $Q$ denote the field of rational numbers. We remind the reader of the following concepts. For $a / b \in Q$ we can write, for a given prime $p, a / b=p^{\alpha} a^{\prime} / b^{\prime}$ where $a^{\prime}$ and $b^{\prime}$ are not divisible by $p$ and where $\alpha$ is an integer. The $p$-adic valuation of $a / b$ is $|a / b|_{p}=p^{-\alpha}$. The completion of $Q$ under this valuation is the $p$-adic number field $Q_{p}$ which is analogous to the field of real numbers which is the completion of $Q$ under the ordinary absolute value. The analogue of the field of complex numbers is obtained by taking the algebraic closure of $Q_{p}$ and then completing this field under the extension of the $p$-adic valuation. We denote this field by $\Omega_{p}$. It is now possible to consider analytic functions over $\Omega_{p}$. If a function $f(z)$ is given by a power series with coefficients which are in the complex field and $\Omega_{p}$, that is, if $f^{(n)}(0)$ is in both fields for $n=0,1, \cdots$, then $f(z)$ may be considered to be simultaneously a function of a complex and a $p$-adic variable. For

Received by the editors May 24, 1967. 
instance the coefficients could be rational. In fact, it is meaningful to speak of an algebraic number as belonging to both $\Omega_{p}$ and the complex field. Indeed let $\alpha$ be algebraic. The minimal equation for $\alpha$ may factor over $Q_{p}$. Let $a_{0} x^{n}+\cdots+a_{n}$ be an irreducible factor over $Q_{p}$ of the minimal equation where $a_{0}, \cdots, a_{n}$ are relatively prime integers in $Q_{p}$. Then the equation $a_{0} x^{n}+\cdots+a_{n}=0$ has a solution in $\Omega_{p}$. One of these solutions is identified with $\alpha$ and the extension of the $p$-adic valuation on $Q$ gives

$$
|\alpha|_{p}=\left|a_{n} / a_{0}\right|_{p}^{1 / n} \text {. }
$$

Let $|a|_{\infty}$ denote the ordinary absolute value of the number $a$.

Let

$$
f_{\mu}(z)=\sum_{i=0}^{\infty} a_{i \mu} z^{i} \quad(\mu=1, \cdots, m)
$$

be $m$ functions of $a$ complex and $p$-adic variable $z$ where each $a_{i \mu}$ is algebraic. Let $\left\{z_{\nu}\right\}$ be a sequence of distinct algebraic points. Assume that

$$
\lim _{\nu \rightarrow \infty}\left|z_{\nu}\right|_{v}=0
$$

for $V=\infty, p$. For example we could have $z_{\nu}=\nu ! / p_{\nu !}$ where $p_{i}$ denotes the $i$ th prime. Suppose that $f_{\mu}\left(z_{v}\right)$ is the same algebraic number regardless of which field $z_{\nu}$ is in. For some index $\nu_{0}$ let

$$
P_{\nu}(V)=\left|z_{\nu_{0+\nu+1}}-z_{\nu_{0}+1}\right|_{\nu} \cdots\left|z_{\nu_{0}+\nu+1}-z_{\nu_{0}+\nu}\right|_{V}
$$

and

$$
\rho(V)=\liminf _{\nu \rightarrow \infty} \frac{\log \left|\log P_{\nu}(V)\right|_{\infty}}{\log \nu} .
$$

Then $\rho(V)$ satisfies $1 \leqq \rho(V) \leqq \infty$ and is a measure of the rate of convergence of the sequence $\left\{z_{\nu}\right\}$; the more rapidly the sequence converges, the larger $\rho(V)$. Let

$$
\gamma_{\mu}=\limsup _{\nu \rightarrow \infty} \frac{\log \log 3 H\left(f_{\mu}\left(z_{\nu}\right)\right)}{\log \nu}, \quad \prod_{\mu=1}^{m} \operatorname{deg} f_{\mu}\left(z_{\nu}\right) \leqq h_{\nu}
$$

with $h_{\nu}$ nondecreasing. Let

$$
D_{r}=\sum_{x=\nu_{0}+1}^{\nu_{0}+\gamma} h_{x}, \quad \operatorname{deg} Q\left(f_{1}\left(z_{\nu_{0+\nu}}\right), \cdots, f_{m}\left(z_{p_{0+\gamma}}\right)\right) \leqq d_{\nu}
$$

and 


$$
\alpha=\limsup _{\nu \rightarrow \infty} \frac{\log \left(D_{\nu}^{1 / m} d_{\nu+1}\right)}{\log \nu} .
$$

THEOREM 1. Under the above conditions the functions $f_{1}(z), \cdots, f_{m}(z)$ are algebraically dependent if they have a positive complex and p-adic radius of convergence and if

$$
\alpha+\frac{1}{m} \sum_{\mu=1}^{m} \gamma_{\mu}<\max _{V=\infty, p} \rho(V) .
$$

Remark. Two sequences $z_{\nu}(\infty), z_{\nu}(p)$ of possibly transcendental complex and $p$-adic numbers may be used. In which case $\alpha, f_{\mu}\left(z_{\nu}(V)\right)$ and $\gamma_{\mu}$ would depend on $V$, condition (2) is replaced by

$$
\max _{V=\infty, p}\left(\alpha(V)+\frac{1}{m} \sum_{\mu=1}^{m} \gamma_{\mu}(V)\right)<\max _{V=\infty, p} \rho(V),
$$

and $h_{\chi}$ is replaced by $\max _{V} h_{\chi}(V)$.

Proof. The proof is somewhat similar to arguments in the paper [2] so we shall only outline the details.

Let

$$
\phi(z)=\sum_{\tau_{\mu=0}}^{t_{\mu}} c_{\tau_{1} \cdots \tau_{m}} f_{1}^{\tau_{1}}(z) \cdots f_{m}^{\tau_{m}}(z), \quad(\mu=1, \cdots, m) .
$$

We shall show that for suitably chosen integers $t_{\mu}$ there exists integers $c_{\tau_{1} \cdots \tau_{m}}$ not all zero and not too large so that $\phi(z) \equiv 0$. Consider the $n$ equations

$$
\phi(z)=0, \quad z=z_{\nu_{0+1}}, \cdots, z_{v_{0}+n},
$$

in $c_{\tau_{1} \cdots \tau_{m}}$. Let $\beta_{\mu}=\beta_{\mu}(\nu)=f_{\mu}\left(z_{\nu}\right), \quad \delta(\mu)=\delta(\mu, \nu)=\operatorname{deg} \beta_{\mu}$ and $b_{\hat{o}(\mu)}$ $=b_{\delta(\mu)}(\nu)$ be the denominator of $\beta_{\mu}$ for $\mu=1, \cdots, m$. Then the coefficients in (4) can be expressed as

$$
\begin{aligned}
\beta_{1}^{\tau_{1}} \cdots \beta_{m}^{\tau_{m}}=b_{\delta(1)}^{-\tau_{1}} & \cdots b_{\delta(m)}^{-\tau_{m}} \\
& \left.\cdot \sum_{\sigma_{\mu=0}(\mu)-1}^{\delta\left(\tau_{1}\right.}, \cdots, \tau_{m}, \sigma_{1}, \cdots, \sigma_{m}\right) \beta_{1}^{\sigma_{1}} \cdots \beta_{m}^{\sigma_{m}}
\end{aligned}
$$

where the $a$ 's are integers which satisfy

$$
\left|a\left(\tau_{1}, \cdots, \tau_{m}, \sigma_{1}, \cdots, \sigma_{m}\right)\right|_{\infty} \leqq \prod_{\mu=1}^{m}\left(2 H\left(\beta_{\mu}\right)\right)^{\tau_{\mu}}
$$

If we substitute (5) into (4) and set equal to zero the resulting coefficients of $\beta_{1}^{\tau_{1}} \cdots \beta_{m}^{\tau_{m}}$ we obtain 


$$
\sum_{\tau_{\mu}=0}^{t_{\mu}} b_{\delta(1)}^{t_{1}-\tau_{1}} \cdots b_{\delta(m)}^{t_{m}-\tau_{m}} a\left(\tau_{1}, \cdots, \tau_{m}, \sigma_{1}, \cdots, \sigma_{m}\right) c_{\tau_{1} \cdots \tau_{m}}=0
$$

for $\nu=\nu_{0}+1, \cdots, \nu_{0}+n ; \sigma_{\mu}=0, \cdots, \delta(\mu)-1 ; \mu=1, \cdots, m$. Thus we have a system (6) of at most $D_{n}$ equations with integral coefficients in the $\prod_{\mu=1}^{m}\left(t_{\mu}+1\right)$ unknowns $c_{\tau_{1}} \ldots \tau_{m}$. Let

$$
t_{\mu}=\left[\left(2 D_{n}\right)^{1 / m}\left(\nu_{0}+n\right)^{(1 / m) \Sigma_{k-1}^{m} \gamma_{k}-\gamma_{\mu}}\right]
$$

where $[x]$ denotes the greatest integer in $x$. Then the number of unknowns is at least twice the number of equations. The coefficients in (6) have, for a given $\epsilon>0$, absolute value at most

$$
\prod_{\mu=1}^{m}\left\{2 H\left(f_{\mu}\left(z_{\nu}\right)\right)\right\}^{t_{\mu}} \leqq \exp \left(c_{1} D_{n}^{1 / m} n^{(1 / m) \Sigma_{k-1}^{m} \gamma_{k}+\epsilon}\right)
$$

where $c_{1}$ and the $c_{i}$ 's to follow are constants. Thus (6) has a solution in integers $c_{\tau_{1} \ldots \tau_{m}}$, not all zero, for which

$$
\left|c_{\tau_{1} \cdots \tau_{m}}\right|_{\infty} \leqq \exp \left(c_{2} D_{n}^{1 / m} n^{(1 / m) \Sigma_{k-1}^{m} \gamma_{k}+\epsilon}\right) .
$$

Hence (4) holds. We shall now show that if $\phi(z)=0$ for $z=z_{\nu_{0+1}}, \cdots$, $z_{v_{0}+n_{1}}$ then $\phi(z)=0$ for $z=z_{v_{0}+n_{1}+1}$ if $n_{1} \geqq n$ and $n$ is sufficiently large. By applying the maximum principle to the analytic function

$$
\frac{\phi(z)}{\left(z-z_{\nu_{0}+1}\right) \cdots\left(z-z_{\nu_{0}+n_{1}}\right)}
$$

in some small closed disk about the origin we obtain an upper bound for $\left|\phi\left(z_{\nu_{0}+n_{1}+1}\right)\right| v$.

An upper bound for the conjugates can be obtained directly from (3). Let $K$ be the denominator of $\phi\left(z_{\nu_{0}+n_{1}+1}\right)$. Then if we combine these estimates we conclude that the norm of the algebraic integer $K \phi\left(z_{\nu_{0}+n_{1}+1}\right)$ satisfies

$$
\begin{aligned}
\prod_{V=\infty, p} \mid N & \left.\left(K \phi\left(z_{\nu_{0}+n_{1}+1}\right)\right)\right|_{V} \\
& \leqq \exp \left(c_{3} n_{1}^{\alpha+(1 / m) \Sigma_{\mu-1 \gamma}^{m} \gamma_{\mu}+2 \epsilon}+c_{4} n_{1}-\sum_{V} n_{1}^{\rho(V)-\epsilon / 2} \log n_{1}\right) .
\end{aligned}
$$

If $\rho(V) \neq 1$ for some $V$ we see by (2) that the right side of (7) is less than 1 . Thus $\phi\left(z_{v_{0}+n_{1}+1}\right)=0$ for otherwise the left side of $(7)$ would be at least 1 . Thus $\phi(z)$ vanishes at the entire sequence $z_{v_{0+1}}, z_{v_{0+2}}, \cdots$ and hence it must be identically zero. If $\rho(V)=1$ for each $V$, then the term 


$$
\sum_{V} n_{1}^{\rho(V)-\epsilon / 2} \log n_{1}
$$

may be replaced by $c_{5} n_{1}$ with $c_{5}>c_{4}$ and again $\phi(z) \equiv 0$.

If equality holds in (2), then more complicated conditions are required. Here it becomes significant to consider, say, $r$ primes $p_{1}$, $\cdots, p_{r}$. Let $f_{\mu}(z), \mu=1, \cdots, m$, be given by (1) with $a_{i \mu}$ algebraic. Let $z_{\mu}(V)$ be a sequence of distinct points, not necessarily algebraic, for $V=\infty, p_{1}, \cdots, p_{r}$ with $z_{\nu}(\infty)$ complex and $z_{\nu}\left(p_{i}\right)$ in $\Omega_{p_{i}}, i=1$, $\cdots, r$. Suppose that $\lim _{\nu \rightarrow \infty}\left|z_{\nu}(V)\right|_{V}=0$ for $V=\infty, p_{1}, \cdots, p_{r}$ and that $f_{\mu}\left(z_{\nu}(V)\right)$ is algebraic for $\mu=1, \cdots, m ; \nu=1,2, \cdots ; V=\infty, p_{1}$, $\cdots, p_{r}$. For simplicity in the statement of the theorem, we assume that the degree of $f_{\mu}\left(z_{\nu}(V)\right)$ is bounded. Here $f_{\mu}\left(z_{\nu}(V)\right)$ and $\gamma_{\mu}$ depend on $V$. Let

and

$$
\Omega_{\mu}(V)=\limsup _{\nu \rightarrow \infty} \frac{\log H\left(f_{\mu}\left(z_{\nu}(V)\right)\right)}{\nu^{\gamma_{\mu}(V)} \log \nu}
$$

$$
\sigma(V)=\liminf _{\nu \rightarrow \infty} \frac{\left|\log P_{\nu}(V)\right|_{\infty}}{\nu^{\rho(V)} \log \nu} .
$$

Then $\sigma(V)$ is a refinement of the measure $\rho(V)$ of the sequence $\left\{z_{\nu}(V)\right\}$. Let

$$
\operatorname{deg} Q\left(f_{1}\left(z_{\nu}(V)\right), \cdots, f_{m}\left(z_{\nu}(V)\right)\right) \leqq d
$$

for $\nu=1,2, \cdots$ and $V=\infty, p_{1}, \cdots, p_{r}$.

THEOREM 2. Under the above conditions the functions $f_{1}(z), \cdots, f_{m}(z)$ are algebraically dependent if they have a positive complex and $p_{i}$-adic radius of convergence for $i=1, \cdots, r$ and if either (a) or (b) below hold.

(a) $\max _{V}\left(1 / m+(1 / m) \sum_{\mu=1}^{m} \gamma_{\mu}(V)\right)<\max _{V} \rho(V)$.

(b) $1 / m+(1 / m) \sum_{\mu=1}^{m} \gamma_{\mu}(V)=\rho(V)$ for each $V, \rho(\infty)=\rho\left(p_{1}\right)=\ldots$ $=\rho\left(p_{r}\right) \geqq \gamma_{\mu}$, for each $\mu, \Omega_{\mu}(V)<\infty$, for each $V$ and $\mu$ and

$$
4 m d^{2} \sum_{V}\left(2(r+1) \prod_{\mu=1}^{m} \Omega_{\mu}(V)\right)^{1 / m}<\sum_{V} \sigma(V) .
$$

The proof is similar to that of Theorem 1.

\section{REFERENCES}

1. G. Bachman, Introduction to p-adic numbers and valuation theory, Academic Press, New York, 1964.

2. D. L. Hilliker, On analytic functions which have algebraic values at a convergent sequence of points, Trans. Amer. Math. Soc. 126 (1967), 534-550.

UNIVERSity OF CALIFornia at IRVine 